\title{
SEMI-DISCRETE COINCIDENCE IN THE MID-FREQUENCY SOUND TRANSMISSION THROUGH RIB-STIFFENED PANELS
}

\author{
Edwin Reynders, Cédric Van hoorickx and Arne Dijckmans \\ KU Leuven Dept. of Civil Engineering \\ Kasteelpark Arenberg 40, B-3001 Leuven, Belgium \\ edwin.reynders@kuleuven.be
}

\section{Keywords:}

\begin{abstract}
Plates with attached beams or ribs, rather than monolithic plates, are commonly employed in civil and mechanical engineering for reasons of weight and static stiffness. Unfortunately, attaching stiffeners to a plain plate generally increases its radiation efficiency and reduces its airborne sound insulation. This contribution aims at gaining insight into the sound insulation of rib-stiffened plates in the mid-frequency range, where the modal behavior of the plate is still important, but the neighboring sound fields can already be considered as diffuse. A detailed finite element model of a PMMA plate with steel stiffeners attached is constructed and coupled to a reverberant sound field model of the adjoining room(s) within the recently developed hybrid finite element - statistical energy analysis framework. This framework is computationally very efficient, it enables to compute the coupling loss factors between the sound fields in a rigorous, straight forward way, and the uncertainties due to random wave scattering - which is the physical origin of the diffuse field - can be quantified. The finite element model of the plate is first calibrated by minimizing the difference between its lowest natural frequencies and mode shapes and the corresponding measured values. The hybrid model is then employed for predicting the sound reduction index across the building acoustics frequency range. These predictions are validated against airborne sound insulation measurements. Within a wide frequency range, pronounced dips are observed at specific natural frequencies of the plate, at which the wavelength of the corresponding mode shape is close to the free acoustic wavelength. Since there is an obvious connection with the coincidence phenomenon for infinite plates, the observed phenomenon is termed the semi-discrete coincidence phenomenon. It is shown that in the mid-frequency range, an important increase in airborne sound insulation can be achieved by suppressing only a few particular resonances of the considered rib-stiffened plate.
\end{abstract}




\section{INTRODUCTION}

Rib-stiffened plates are commonly employed in civil and mechanical engineering as they achieve a similar strength and stiffness as plain plates with a substantially lower weight. Unfortunately, attaching stiffeners to a plain plate generally increases its radiation efficiency and reduces its airborne sound insulation [3].

Early investigations into these phenomena led to two approximate physical explanations based on infinite plate theory. Firstly, when the wavelength of deformation is much larger than the distance between the stiffeners, the main effect of the stiffeners is to introduce orthotropy into the plate. The effect of the stiffeners is then that the frequency range in which coincidence has a detrimental effect on radiation efficiency and transmission loss is broadly smeared out towards lower frequencies when compared to the plane plate [6]. Secondly, when the wavelength of deformation is much smaller than the distance between the stiffeners, the stiffeners have the approximate acoustic effect of dividing the plate into smaller panels, the radiation efficiency of which is much larger than the radiation efficiency of the large unstiffened plate [12]. These investigations were followed by several more refined analyses; see, e.g., [1, 2, 5, 11, 13].

The present contribution aims at gaining additional insight into the sound transmission through finite rib-stiffened plates by investigating the behavior of the room-wall-room system in the midfrequency range. This is the frequency range in which the sound fields in the rooms can already be considered as diffuse (in contrast to the low-frequency range), while the modal behavior of the wall is still important (in contrast to the high-frequency range). A detailed finite element model of the rib-stiffened plate is constructed and coupled to a reverberant model of the adjoining rooms within the recently developed hybrid finite element - statistical energy (FE-SEA) analysis framework $[16,10,15]$. This framework is substantially more flexible and general than conventional statistical energy analysis as it allows, for example, (i) incorporating diffuse and non-diffuse vibro-acoustic system components within a single model in a computationally efficient way, (ii) assessing the uncertainty inherent in the assumption of diffuse sound fields in the rooms, and (iii) computing coupling loss factors in a rigorous and straightforward way, accounting automatically for both resonant and non-resonant transmission [9].

As a specific case, a polymethyl methacrylate (PMMA) plate with steel stiffeners attached is considered. By analysis of the model predictions, it is found that the finite dimensions of the plate result in a semi-discrete coincidence phenomenon, i.e., the wavelength of a mode shape of the rib-stiffened plate is close to the free acoustic wavelength at the corresponding natural frequency. This is seen to result in an oscillating narrow-band transmission loss over a wide frequency range, which is in contrast to the discrete coincidence phenomenon for finite homogenous isotropic plates $[4,7]$. Only a few of the plate modes in this wide frequency range exhibit semi-discrete coincidence, which suggests that the sound insulation in that frequency range can be substantially increased by suppressing only a few structural modes.

The reminder of this article is structured as follows. In section 2, the dynamic structural model of the stiffened plate is discussed in detail. In section 3, the finite element model is incorporated in a hybrid FE-SEA model of the transmission suite and the transmission loss of the rib-stiffened plate is computed and validated against measurements. A physical interpretation in terms of semi-discrete coincidence is given in section 4, while the potential of suppression a few particular modes is investigated in section 5. Conclusions are drawn in section 6. 


\section{FINITE ELEMENT MODEL OF THE STIFFENED PLATE}

The example structure that is considered in this paper consists of a base plate made of PMMA, to which 11 steel L30 stiffeners are attached. The base plate has a width of $L_{\mathrm{x}}=$ $1.25 \mathrm{~m}$, a height of $L_{\mathrm{y}}=1.5 \mathrm{~m}$ and a thickness of $t_{\mathrm{p}}=15 \mathrm{~mm}$. The steel L-shaped stiffeners have an outer leg length of $L_{\mathrm{s}}=30 \mathrm{~mm}$ and a thickness of $t_{\mathrm{s}}=3 \mathrm{~mm}$. The center-to-center spacing between the stiffeners is $d_{\mathrm{x}}=100 \mathrm{~mm}$, the distance between a vertical edge of the plate and a vertical edge of the closest stiffener is $a_{\mathrm{x}}=110 \mathrm{~mm}$, and the distance between a horizontal edge of the plate and the closest end section of a stiffener is $a_{\mathrm{y}}=52.5 \mathrm{~mm}$. The stiffeners are both glued to the base plate over their entire length, and additionally screwed to the base plate at four points.

A finite element (FE) model of this structure was made using the ANSYS software package. Both the base plate and the steel stiffeners were modeled with four-node linear thin shell elements (of the SHELL181 type). The mesh is shown in Fig. 1. When connecting the plate and the horizontal stiffener legs, the offset of the stiffeners with respect to the midplane of the plate was taken into account. The out-of-plane displacements were restrained at the plate edges. No other boundary restrictions were applied, and the three in-plane rigid body modes were removed from the model. This set of boundary conditions agrees with the dynamic plate boundary conditions that are applied in the sound transmission suite of the KU Leuven Laboratory of Acoustics. For the steel profiles, a Young's modulus of $E_{\mathrm{s}}=210 \mathrm{GPa}$, Poisson's ratio of $\nu_{\mathrm{s}}=0.3$ and density of $\rho_{\mathrm{s}}=7850 \mathrm{~kg} / \mathrm{m}^{3}$ were taken. For the PMMA base plate, a Young's modulus of $E_{\mathrm{p}}=4.564 \mathrm{GPa}$, a Poisson's ratio of $\nu_{\mathrm{p}}=0.35$ and a density of $\rho_{\mathrm{p}}=1170 \mathrm{~kg} / \mathrm{m}^{3}$ were taken.

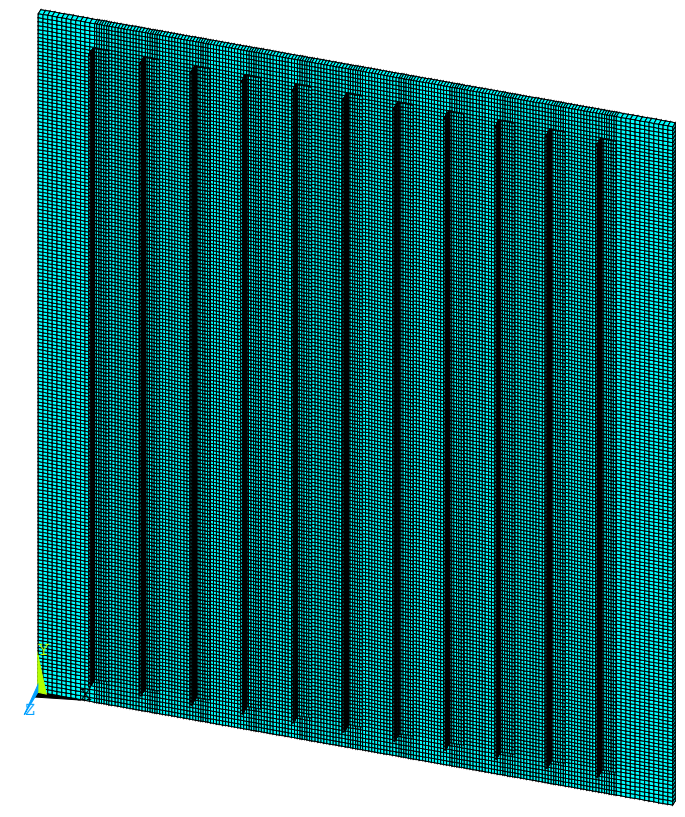

Figure 1: Finite element mesh. The volumetric representation is for visualization purposes only; both the plate and the stiffeners are modelled with two-dimensional shell elements.

\section{SOUND INSULATION PREDICTIONS AND EXPERIMENTAL VALIDATION}

For the sound insulation predictions, a hybrid finite element - statistical energy analysis (FESEA) approach $[10,15,16]$ is adopted. This approach allows coupling diffuse field (or SEA 


\begin{tabular}{|c|c||c|c||c|c||c|c|}
\hline$f[\mathrm{~Hz}]$ & $\eta[-]$ & $f[\mathrm{~Hz}]$ & $\eta[-]$ & $f[\mathrm{~Hz}]$ & $\eta[-]$ & $f[\mathrm{~Hz}]$ & $\eta[-]$ \\
\hline 100 & 0.049 & 250 & 0.046 & 630 & 0.060 & 1600 & 0.044 \\
125 & 0.049 & 315 & 0.056 & 800 & 0.055 & 2000 & 0.038 \\
160 & 0.056 & 400 & 0.062 & 1000 & 0.059 & 2500 & 0.035 \\
200 & 0.065 & 500 & 0.040 & 1250 & 0.054 & 3150 & 0.027 \\
\hline
\end{tabular}

Table 1: Damping loss factor measured in $1 / 3$ octave bands.

subsystem) models of modally dense components to deterministic models of modally sparse components in a rigorous way. A diffuse field model is a probabilistic wave field model, and therefore the predicted sound transmission loss is also a probabilistic quantity.

The model of the considered transmission suite is divided into three components: emitting room, partition wall, and receiving room. The rooms each have a volume of about $87 \mathrm{~m}^{3}$ and a reverberation time of about $1.5 \mathrm{~s}$. They are modeled to carry a diffuse wave field, as SEA subsystems. The values $c=343 \mathrm{~m} / \mathrm{s}$ and $\rho_{\mathrm{a}}=1.2 \mathrm{~kg} / \mathrm{m}^{3}$ are adopted for the sound speed and air density, respectively. For the partition wall, which consists of the baffled rib-stiffened plate, the finite element model of section 2 is employed. Measured values are used for the damping loss factor of the wall as reported in Table 3. Full details on sound transmission modeling with the hybrid method can be found in [15]. For completeness, it is mentioned that the acoustic direct field dynamic stiffness matrices of the rooms (SEA subsystems) were evaluated by means of a wavelet approach [8], using a square interpolation grid covering the flat plate surface and a distance between the grid points of $2.08 \mathrm{~cm}$.

Fig. 2 displays the model predictions and compares these with narrow-band (1/48 octave) sound reduction index measurements, which have been performed in the transmission suite of the KU Leuven Laboratory of Acoustics. Not only the mean of the predicted sound transmission loss values are plotted, but also the related $\pm 2 \sigma$ confidence intervals, which correspond to $95 \%$ confidence intervals when a Gaussian probability distribution can be assumed. One should keep in mind that this uncertainty is due to the assumption of a harmonic diffuse field model for the rooms. This assumption can be justified when the acoustic pressure field in the rooms is sensitive to small wave scattering elements, and for the transmission rooms at KU Leuven this is the case from about $140 \mathrm{~Hz}$ onwards [15]. At lower frequencies, the uncertainty due to the presence of small wave scattering elements will be overestimated with a diffuse field model. Furthermore, individual room resonances, which are absent in the adopted hybrid model, will be important at such frequencies. The present analysis however is concerned with mid-frequency sound transmission.

The critical frequency of the infinite panel without stiffeners would be at $2051 \mathrm{~Hz}$. The critical frequency of the ribbed plate in the weak bending direction (i.e., for bending around an axis parallel to the ribs) can be estimated at $2735 \mathrm{~Hz}$ by neglecting the stiffness of the steel ribs but accounting for their mass. The critical frequency in the strong bending direction can be estimated at $461 \mathrm{~Hz}$ by taking both the stiffness and the mass of the ribs into account. Between both frequencies, a broad coincidence zone exists for infinite plates. For the finite plate that is studied here, a strong oscillation of transmission loss with frequency is observed in this zone. Such oscillation is not predicted by, and cannot be explained from, infinite plate theory. It is therefore investigated with a more refined model in the next sections.

A very good agreement between the measurements and the hybrid model predictions is observed in the frequency range in which the model is valid (above $140 \mathrm{~Hz}$ ). The oscillation 

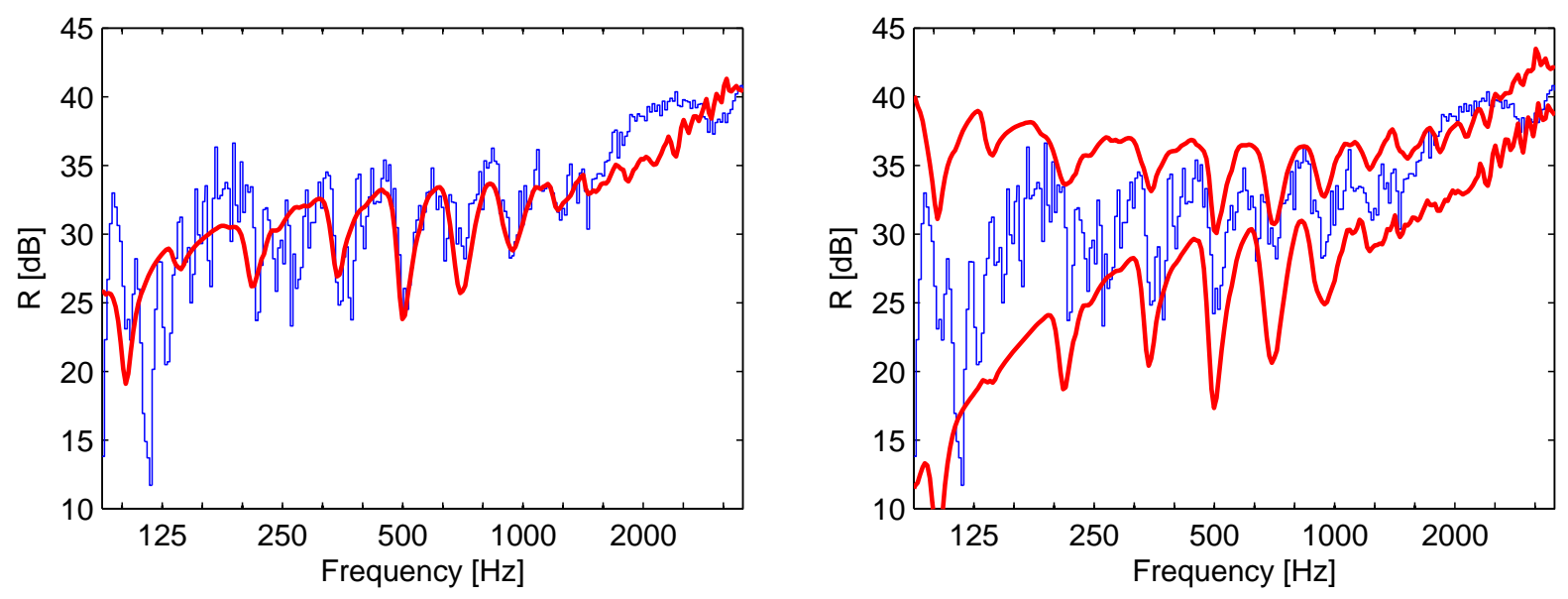

Figure 2: Measured sound reduction index of a rib-stiffened plate in 1/48-octave bands (blue) vs. the harmonic mean values (red, left figure) and $95 \%$ confidence intervals (red, right figure) as predicted with the hybrid FE-SEA method.

of the transmission loss is very well predicted, and the measurements fall almost completely within the $95 \%$ confidence interval which represents the uncertainty caused by the diffuse field assumption for the sound fields in the rooms.

\section{SEMI-DISCRETE COINCIDENCE}

The pronounced dips that are observed in both the measured and the predicted sound reduction index above $140 \mathrm{~Hz}$ correspond with some of the eigenfrequencies of the finite-sized ribstiffened plate. They do not correspond with eigenfrequencies of the bare rooms, as these are not included in the hybrid FE-SEA model. In order to get more insight into why certain eigenfrequencies of the plate result in a pronounced reduction of the airborne sound insulation while others do not, both the in-vacuo modes of the rib-stiffened plate (as determined from the finite element model) and the acoustic dispersion curve are plotted in the frequency-wavenumber domain (Fig. 3). The structural wavenumber of mode $j$ was computed as the amplitude of $\left[k_{\mathrm{x} j}, k_{\mathrm{y} j}\right]$, where $k_{\mathrm{x} j}$ denotes the horizontal and $k_{\mathrm{y} j}$ the vertical wavenumber. $k_{\mathrm{x} j}$ and $k_{\mathrm{y} j}$ are determined through counting the number of approximate half sine wavelengths in the horizontal and vertical directions, respectively, of mode $j$.

This counting process was automated as follows. First, the correlation between a computed mode shape and all possible mode shapes that consist of between 0 and 15 half sine wavelengths in both horizontal directions was determined by computing the MAC value [14]. The combination that yielded the highest MAC value was then retained for determining the wavenumber corresponding to the mode shape. In Fig. 3, a different symbol is used for wavenumbers that are computed based on a high MAC value and those based on a low MAC value.

Subsequently, the frequency-wavenumber relation (also called dispersion curve) for sound waves was plotted in the same figure. It can be seen that this dispersion curve comes close to a mode of the rib-stiffened plate at $212 \mathrm{~Hz}, 345 \mathrm{~Hz}, 502 \mathrm{~Hz}, 693 \mathrm{~Hz}$, and $964 \mathrm{~Hz}$, which are precisely the frequencies at which the sound transmission loss of the plate dips. The dips can therefore be attributed to a semi-discrete coincidence phenomenon which involves only specific plate modes, namely, those modes for which the mode shapes exhibit one half wavelength in the horizontal direction. From around $1000 \mathrm{~Hz}$, many plate modes match closely the acoustic wavelength at their resonance frequency, and pronounced dips in the sound reduction index are 


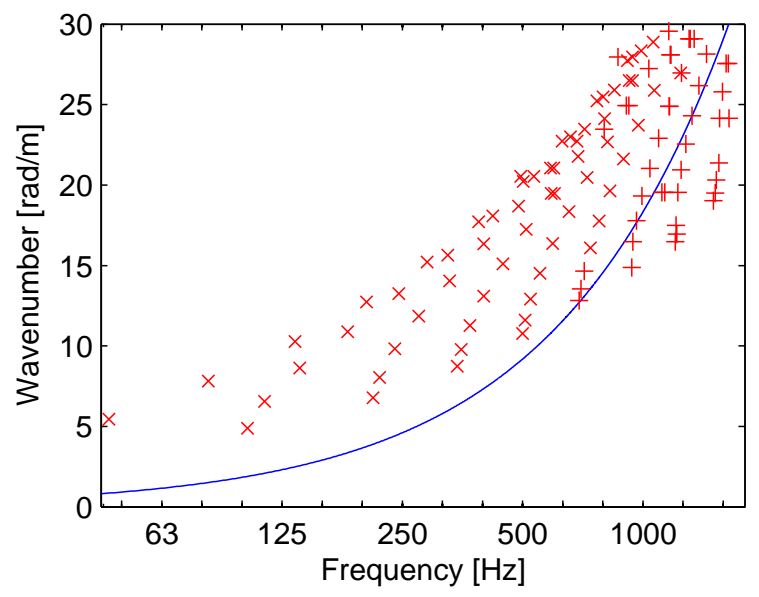

Figure 3: Modes of the rib-stiffened plate as predicted with the updated finite element model (crosses) in the frequency-wavenumber domain, together with the frequency-wavenumber relation for sound waves in an unbounded domain (solid lines). x: MAC value between the mode shape and the closest half-sines mode shape is higher than 0.7. +: MAC value is lower than 0.7 .

no longer observed.

\section{INFLUENCE OF MODE SUPPRESSION}

In the light of this physical interpretation, it is instructive to investigate by how much the sound insulation of the plate improves when these five modes are suppressed. Therefore, the sound transmission loss of the plate is re-computed but without taking these five modes into account. The sound insulation improvement that is computed in this way is an upper bound, because in reality mode suppression measures - such as local stiffening, attachment of tuned mass dampers, and active vibration control - are never perfect.
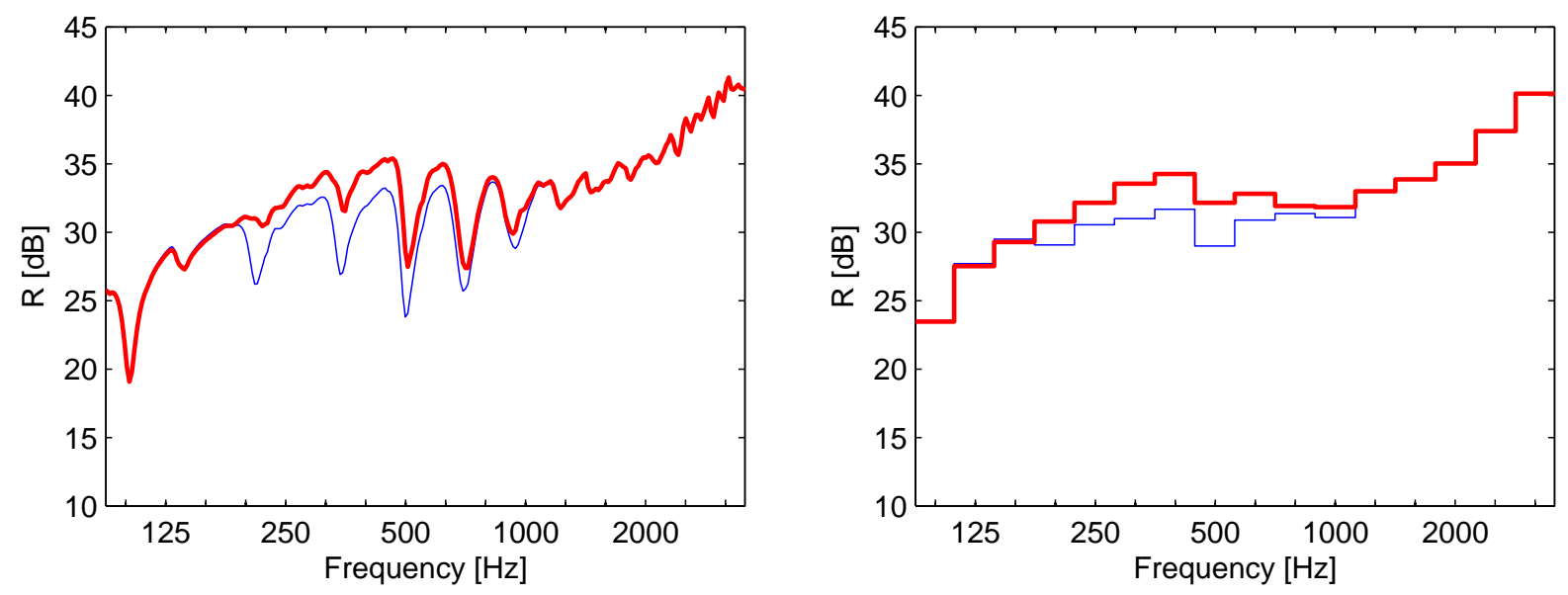

Figure 4: Predicted sound transmission loss of the rib-stiffened plate with (red) and without (blue) mode suppression. Left: harmonic values, right: 1/3 octave band averages.

Fig. 4 compares the computed sound transmission loss with and without mode suppression. Both harmonic values and one-third octave band averages are shown. Suppressing the modes with natural frequencies of $212 \mathrm{~Hz}, 345 \mathrm{~Hz}$ has the largest influence: the dips in the transmission loss are largely reduced, resulting in an increase of the transmission loss with about $5 \mathrm{~dB}$ around 
those frequencies. Suppressing the modes at $502 \mathrm{~Hz}, 693 \mathrm{~Hz}$ and $964 \mathrm{~Hz}$ has a smaller effect. This can be understood from Fig. 3: around these frequencies, the bending wavelengths of several other modes also match the acoustic wavelength well, and as a result, suppressing only one of these modes is not effective anymore.

\section{CONCLUSIONS}

In this paper, valuable insight into the airborne sound insulation of finite-sized rib-stiffened plates was obtained by constructing a detailed finite element model of the structure and coupling this to diffuse field models for the adjoining rooms within the computationally efficient hybrid FE-SEA framework. For the specific rib-stiffened plate that was considered here, a pronounced oscillation in the narrow-band sound transmission loss was observed over a broad frequency range. The oscillation dips correspond to only a few natural frequencies of the plate, at which the wavelength of the corresponding mode shape is close to the acoustic wavelength, resulting in a semi-discrete coincidence phenomenon. This observation is important with respect to vibration control because within this broad frequency range, an increase in the airborne sound insulation can theoretically be achieved by suppressing only a few particular plate resonances.

\section{REFERENCES}

[1] J.S. Anderson and M. Bratos-Anderson. Radiation efficiency of rectangular orthotropic plates. Acta Acustica united with Acustica, 91(1):61-76, 2005.

[2] J. Brunskog. Near-periodicity in acoustically excited stiffened plates and its influence on vibration, radiation and sound insulation. Acta Acustica united with Acustica, 90(2):301$312,2004$.

[3] F. Fahy and P. Gardonio. Sound and structural vibration. Academic Press, Oxford, UK, 2nd edition, 2007.

[4] L. Gagliardini, J. Roland, and J.L. Guyader. The use of a functional basis to calculate acoustic transmission between rooms. Journal of Sound and Vibration, 145(3):457-478, 1991.

[5] C. Guigou-Carter and M. Villot. Modelling of sound transmission through lightweight elements with stiffeners. Building Acoustics, 10(3):193-209, 2003.

[6] M. Heckl. Untersuchungen an orthotropen Platten. Acustica, 10:109-115, 1960.

[7] R. Josse and C. Lamure. Transmission du son par une paroi simple. Acustica, 14(5):266$280,1964$.

[8] R.S. Langley. Numerical evaluation of the acoustic radiation from planar structures with general baffle conditions using wavelets. Journal of the Acoustical Society of America, 121(2):766-777, 2007.

[9] R.S. Langley and J.A. Cordioli. Hybrid deterministic-statistic analysis of vibro-acoustic systems with domain couplings on statistical components. Journal of Sound and Vibration, 321(3-5):893-912, 2009. 
[10] R.S. Langley and V. Cotoni. Response variance prediction for uncertain vibro-acoustic systems using a hybrid deterministic-statistical method. Journal of the Acoustical Society of America, 122(6):3445-3463, 2007.

[11] J.-H. Lee and J. Kim. Analysis of sound transmission through periodically stiffened panels by space-harmonic expansion method. Journal of Sound and Vibration, 251(2):349-366, 2002.

[12] G. Maidanik. Response of ribbed panels to reverberant acoustic fields. Journal of the Acoustical Society of America, 34(6):809-826, 1962.

[13] D.J. Mead. Plates with regular stiffening in acoustic media: vibration and radiation. Journal of the Acoustical Society of America, 88(1):391-401, 1990.

[14] E. Reynders. System identification methods for (operational) modal analysis: review and comparison. Archives of Computational Methods in Engineering, 19(1):51-124, 2012.

[15] E. Reynders, R.S. Langley, A. Dijckmans, and G. Vermeir. A hybrid finite element statistical energy analysis approach to robust sound transmission modelling. Journal of Sound and Vibration, 333(19):4621-4636, 2014.

[16] P.J. Shorter and R.S. Langley. Vibro-acoustic analysis of complex systems. Journal of Sound and Vibration, 288(3):669-699, 2005. 